United Nations Educational Scientific and Cultural Organization

and

International Atomic Energy Agency

THE ABDUS SALAM INTERNATIONAL CENTRE FOR THEORETICAL PHYSICS

\title{
ELASTIC PROPERTIES OF DENSE SOLID PHASES OF HARD CYCLIC PENTAMERS AND HEPTAMERS IN TWO DIMENSIONS
}

\author{
K.W. Wojciechowski ${ }^{1}$ \\ Institute of Molecular Physics, Polish Academy of Sciences, \\ Smoluchowskiego 17, 60-179 Poznań, Poland, \\ K.V. Tretiakov \\ Institute of Molecular Physics, Polish Academy of Sciences, \\ Smoluchowskiego 17, 60-179 Poznań, Poland \\ and \\ The Abdus Salam International Centre for Theoretical Physics, Trieste, Italy \\ and \\ M. Kowalik \\ Institute of Molecular Physics, Polish Academy of Sciences, \\ Smoluchowskiego 17, 60-179 Poznań, Poland.
}

MIRAMARE - TRIESTE

February 2003

$\overline{{ }^{1} \mathrm{kww} @ m a n . p o z n a n . p l}$ 


\begin{abstract}
Systems of model plannar, non-convex, hard-body 'molecules' of five-fold and seven-fold symmetry axes are studied by constant pressure Monte Carlo simulations with variable shape of the periodic box. The molecules, referred to as pentamers (heptamers) are composed of five (seven) identical hard discs-'atoms' with centers forming regular pentagons (heptagons) of sides equal to the disc diameter. The elastic compliances of defect-free solid phases are computed by analysis of strain fluctuations and the reference (equilibrium) state is determined within the same run in which the elastic properties are computed. Results obtained by using pseudo-random number generators based on the idea proposed by Holian and co-workers [B. L. Holian et al., Phys. Rev. E50, 1607 (1994)] are in good agreement with the results generated by DRAND48. It is shown that singular behavior of the elastic constants near close packing is in agreement with the free volume approximation; the coefficients of the leading singularities are estimated. The simulations prove that the highest density structures of heptamers (in which the molecules cannot rotate) are auxetic, i.e. show negative Poisson ratios.
\end{abstract}




\section{INTRODUCTION}

Hard-core systems have been extensively studied in the literature to model various structures of matter [1-34]. The interest in the systems comes from the fact that they can reproduce short range molecular correlations and excluded volume effects. The hard-core systems are also attractive from the point of view of the fundamental requirements of the thermodynamics since, for obvious reasons, they cannot collapse at any pressure. It is worth to add that the free energy of systems interacting through purely hard potentials, infinite when overlaps occur and zero otherwise, further referred to as hard-body systems, is reduced to the product of the temperature and the entropy. (It is so because all available configurations have the same, usually assumed to be zero, potential energy.) In consequence, the complete equation of state of the hard-body systems can be obtained from a single isotherm.

Although the most common application of the hard-body systems concerns the theory of fluids, they can also play the role of very simple (purely geometric) models of solids. In particular, they may help to understand the influence of the molecular shape on the elastic properties of (extremely) anharmonic solids. Determination of elastic properties of hard-body solids is, however, a non-trivial task because of non-analytic character of their interactions. In the past, the elastic properties of hard-body solid systems have been determined for convex bodies only: for hard spheres [35-44], for hard discs [45-50], and for hard ellipsoids [51]. Since most of real molecules are neither spherical nor ellipsoidal, calculations of elastic properties of other systems consisted of anisotropic hard bodies are of interest. In particular, studies of systems containing model molecules of non-convex shapes, which differ qualitatively from convex ones, may reveal new properties and/or phenomena, some of which may find practical applications.

The plannar hard cyclic pentamer and the plannar hard cyclic heptamer (see Fig.1), further referred to as the pentamer and the heptamer, are non-convex, star-shaped [19], hard-body model molecules of 5 -fold and 7 -fold symmetry axes, respectively. These are the lowest symmetry axes forbidden in periodic crystalline phases. Theoretical and experimental studies of the pentamers [52$57]$ and the heptamers $[55,57,58]$ showed that various solid phases can exist in these systems. (We avoid using the word crystal in the context of plannar systems because of the well known problems with the translational order in two dimensions [59,60].) Some thermodynamic and structural properties of these phases have been determined by Monte Carlo simulations [53-58]. In particular, preliminary studies of some dense structures of the heptamers have indicated that they exhibit anomalous (negative) Poisson ratios [55, 58].

The Poisson ratio, $\nu$, is a quantity characterizing deformations of elastic media. It can be determined by introducing an infinitesimal change of the stress along a certain direction (whereas other components of the stress tensor are kept fixed) and measuring the strain along this direction and a direction perpendicular to it. $\nu$ is defined as the negative ratio of the transverse strain change 
to the longitudinal strain change and, in general, it depends on both the directions.

The Poisson ratios of typical materials are non-negative, i.e. the materials contract (expand) transversely when pulled (pushed) longitudinally. It should be stressed, however, that negative Poisson ratio is allowed by the stability conditions even in the case of isotropic systems [61]. Moreover, systems of negative Poisson ratio are not just theoretical curiosities [62,63] but can have a lot of interesting applications. Such systems have been manufactured more than a decade ago [64] and since that time they have been a subject of very intensive studies [65-99]. Systems of negative Poisson ratio have been coined auxetics by Evans [74]. For anisotropic systems showing the negative Poisson ratio in certain directions only, the name axial auxetics has been introduced [89].

The main aim of this paper is to determine elastic properties of the defect-free, dense solid structures of pentamers and heptamers (i.e. in absence of vacancies, dislocations, disclinations, etc.) by applying the strain-fluctuation method [45, 57, 100-103] and to test the convergence of this method for elastically anisotropic phases. The present study resolves the problem of auxeticity of the most dense structures of the heptamers. It extends also the amount of available data concerning systems consisted of anisotropic model molecules interacting through highly anharmonic potentials. Such data are useful to construct various theoretical approximations and to test them.

The structure of the paper is as follows. In Sec. II some basic facts concerning the elasticity theory are recalled and the simulation method is presented. In Sec. III we briefly describe the dense structures of the pentamers and heptamers which elastic properties are studied in the present paper. In Sec. IV the obtained results concerning the elastic properties of the studied structures are discussed and compared with the free volume theory. The last section contains the summary and conclusions.

\section{THE SIMULATION METHOD}

Various methods have been proposed in the literature for simulations of elastic properties of model systems [38, 39, 43, 45, 47, 48, 63, 81, 100-109]. The computations described in the present work were performed by the constant pressure Monte Carlo method with variable shape of the box [110]. The elastic constants were determined by a version [57, 103] of the strain-fluctuation method [100-102]. Although the method seems to be slower convergent than some methods with fixed shape of the periodic box [105, 109], this disadvantage is, in our opinion, fully compensated by simplicity of the method and the fact that all the elastic constants are calculated within a single run during which also the (equilibrium) reference state is determined. (As the determination of the reference state with the same precision in a separate run would require a run of a comparable length, this saves about 50 percent of the simulation time.) The elastic constants can be obtained in a single run also by some other methods $[43,47,104]$ for which, however, as well as for most of other known methods, using of explicit microscopic formulae for the pressure (or even for its 
derivatives $[43,104])$ is required. In the case of anisotropic and non-central molecular interactions the microscopic pressure is, however, often expressed by rather complicated formulae. Applying the present method, one avoids differentiating the interaction potential as well as deriving and implementing to the program the microscopic formulae for the pressure (or the elastic constants). One can notice that the same is true for a method $[44,50]$ which is based on numerical differentiation of the free energy with respect to the strain. (The free energy can be obtained, e.g., by the Frenkel-Ladd method [44,50,111].) In the latter method, however, various deformations around the reference state have to be considered, and the free energy has to be calculated at each of the deformations. In general, other methods require also determination of the reference state in advance, i.e. before the run in which the data necessary to determination of the elastic constants are obtained.

Describing the applied simulation technique requires recalling some general definitions concerning elasticity of solids. This is done in the following subsection. Statistical mechanical basis of the simulation technique is sketched briefly in the second subsection. In the third subsection, the way in which one avoids calculating the strain components during the simulations (and, hence, the necessity to know the reference state in advance) is shown. In the last (fourth) subsection, the remaining details of the performed simulations are presented.

\section{A. Elasticity of solids under external pressure}

The free energy change, $\Delta F_{\text {elastic }}$, corresponding to a thermodynamically reversible elastic deformation of a solid under an external stress $\sigma_{i j}$ can be expanded in power series of the components of the strain tensor. The second order expansion reads [112]

$$
\Delta F_{\text {elastic }} / V_{\text {ref }}=\sum_{i j}^{D} \sigma_{i j} \varepsilon_{i j}+\frac{1}{2} \sum_{i j k l}^{D} C_{i j k l} \varepsilon_{i j} \varepsilon_{k l},
$$

where $V_{r e f}$ is the volume of the reference state, $D$ is the system dimensionality, $C_{i j k l}$ are the components of the tensor of elastic constants (invariant with respect to the following replacements of the indices: $i \leftrightarrow j, k \leftrightarrow l, i, j \leftrightarrow k, l)$,

$$
\varepsilon_{i j} \equiv\left(\partial_{i} u_{j}+\partial_{j} u_{i}+\sum_{k} \partial_{i} u_{k} \partial_{j} u_{k}\right) / 2
$$

is the (Lagrange) strain tensor, $u_{i} \equiv x_{i}-X_{i}$ is the displacement vector, $X_{i}, x_{i}$ describe the reference state and the deformed state, respectively [61], and $\partial_{i} \equiv \frac{\partial}{\partial X_{i}}$.

At constant pressure $p$ (i.e. when the stress is isotropic, $\sigma_{i j}=-p \delta_{i j}$ ) it is, however, more convenient to use other elastic constants, $B_{i j k l}$, obtained by the following free enthalpy (Gibbs free energy) expansion [82]

$$
\Delta G / V_{p} \equiv \Delta\left(F_{\text {elastic }}+p V\right) / V_{p}=\frac{1}{2} \sum_{i j k l}^{D} B_{i j k l} \varepsilon_{i j} \varepsilon_{k l},
$$


where $V_{p} \equiv V_{\text {ref }}$ is the volume of the reference state chosen as the equilibrium state at the pressure $p$, and $V$ is the volume of the deformed system. It can be seen that the second order expansion of the free enthalpy at non-zero pressure is a quadratic form, without linear terms, in the strain components. This is in contrast to the free energy expansion (1) which contains linear terms in the strain components.

There is a simple relation between the elastic constants $B_{i j k l}$ and $C_{i j k l}[82,112]$ :

$$
B_{i j k l}=C_{i j k l}-p\left(\delta_{i k} \delta_{j l}+\delta_{i l} \delta_{j k}-\delta_{i j} \delta_{k l}\right)
$$

The elastic constants $B_{i j k l}$ relate the strain components, $\varepsilon_{k l}$, calculated with respect to the reference state (i.e. the equilibrium state at the isotropic pressure, $p$ ) to the changes of the stress tensor components with respect to the state of the isotropic pressure $\left(\Delta \sigma_{i j} \equiv \sigma_{i j}+p \delta_{i j}\right)$

$$
\Delta \sigma_{i j}=\sum_{k, l} B_{i j k l} \varepsilon_{k l}
$$

Inverting (5) one obtains

$$
\varepsilon_{i j}=\sum_{k, l} S_{i j k l} \Delta \sigma_{k l}
$$

where the elastic compliances, $S_{i j k l}$, are related to the elastic constants $B_{i j k l}$ by the equation [113]

$$
\sum_{m, n} S_{i j m n} B_{m n k l}=\left(\delta_{i k} \delta_{j l}+\delta_{i l} \delta_{j k}\right) / 2
$$

If the single component, $\sigma_{\alpha \alpha}$, of the stress tensor corresponding to pure tension/compression in the $\alpha$-direction is changed infinitesimally, by $\Delta \equiv \Delta \sigma_{\alpha \alpha} \rightarrow 0$, and the other components are kept intact, then the strain calculated with respect to the equilibrium reference state is given by

$$
\varepsilon_{i j}=S_{i j \alpha \alpha} \Delta
$$

Thus, the Poisson ratio measured in the direction $\beta$ perpendicular to $\alpha$ is given by

$$
\nu_{\alpha \beta}=-\frac{\varepsilon_{\beta \beta}}{\varepsilon_{\alpha \alpha}}=-\frac{S_{\beta \beta \alpha \alpha}}{S_{\alpha \alpha \alpha \alpha}} .
$$

Taking into account that $S_{x x y y}=S_{y y x x}$, it follows from (9) that

$$
\nu_{x y}=-\frac{S_{x x y y}}{S_{x x x x}}, \nu_{y x}=-\frac{S_{x x y y}}{S_{y y y y}} .
$$

\section{B. The box matrix and the reference state}

In contrast to a fluid, which can be simulated in a periodic box of an arbitrary chosen shape, simulations of a crystal require using a periodic box which fits to the unit cell at the studied 
thermodynamic conditions. This is because any misfit between the periodic lattice generated by the unit cell and the periodic box results in appearance of additional, unwanted, internal stress which usually makes the studied structure thermodynamically unstable. In general, however, neither the shape nor the size of the unit cell are known for studied systems. This problem can be solved by using the Monte Carlo counterpart [110] of the Parrinello-Rahman idea [114], i.e. by allowing the shape and the size of the periodic box (described by additional degrees of freedom) to change in such a way that, at equilibrium, the (average) box fits the equilibrium unit cell corresponding to the conditions studied. In such simulations, which can be performed in the framework of various generalizations of the constant pressure ensemble [45, 101, 102, 109, 114, 115], it is convenient to describe the periodic box by the box matrix, $\boldsymbol{h}$, which columns are formed by the components of the vectors describing the periodic box [115]. To avoid rotations of the system, the number of the components of the box matrix should be equal to the number of the independent components of the strain tensor, $D(D+1) / 2$. One of possibilities to fulfill this requirement is to put some restrictions on possible directions of the box edges [110]. Another possibility, adopted in the present paper as more convenient for calculations, is to keep the box matrix symmetric during the simulations [116].

Denoting the matrix of the reference state by $\boldsymbol{H} \equiv \boldsymbol{h}_{r e f}$ (which is taken as the average (equilibrium) box matrix, $\boldsymbol{h}$, at the pressure $p$ ), one can write the strain tensor in the form

$$
\varepsilon \equiv \boldsymbol{\varepsilon}(\boldsymbol{h}, \boldsymbol{H})=\left(\boldsymbol{H}^{-1} \cdot \boldsymbol{h} \cdot \boldsymbol{h} \cdot \boldsymbol{H}^{-1}-\boldsymbol{I}\right) / 2
$$

where the matrix $\boldsymbol{h}$ is assumed to be symmetric (what implies that $\boldsymbol{H}$ is also symmetric), $\boldsymbol{H}^{-1}$ is the matrix inverse of the reference box matrix, and $\boldsymbol{I}$ is the unit matrix of dimensionality $D$. The volume of the box is equal to the absolute value of the determinant of the box matrix

$$
V(\varepsilon)=|\operatorname{det}(\boldsymbol{h})|
$$

The configurational partition function of $N$ particles, which are closed in a periodic box $\boldsymbol{V}(\varepsilon)$ obtained by a deformation (described by the strain $\varepsilon$ ) of the reference box, $\boldsymbol{V}_{p} \equiv \boldsymbol{V}_{r e f}=\boldsymbol{V}(\mathbf{0})$, can be written as

$$
\begin{aligned}
Z[\boldsymbol{V}(\varepsilon)] & =\exp \left(-\frac{F[\boldsymbol{V}(\varepsilon)]}{k T}\right) \\
& =\frac{1}{N !} \int_{\boldsymbol{V}(\boldsymbol{\varepsilon})} d \boldsymbol{r}^{(N)} \int d \boldsymbol{\Omega}^{(N)} \exp \left(-\frac{U}{k T}\right)
\end{aligned}
$$

where $\boldsymbol{r}_{i}, \boldsymbol{\Omega}_{i}(i=1, \ldots, N)$ denote the particle positions and orientations, $U$ is the interaction energy, $k$ is the Boltzmann constant, $T$ is the temperature, and $F[\boldsymbol{V}(\varepsilon)]$ is the free energy of the system at the strain $\varepsilon$.

For further considerations it is convenient to define the following partition function

$$
Z=\int d \varepsilon^{\frac{D(D+1)}{2}} \exp \left[-\frac{p V(\varepsilon)}{k T}\right] Z[\boldsymbol{V}(\varepsilon)]
$$


where the integration over the strain tensor concerns all its $\frac{D(D+1)}{2}$ independent components. Using (13) and (3) one obtains

$$
\begin{aligned}
Z= & \int d \varepsilon^{\frac{D(D+1)}{2}} \exp \left[-\frac{p V(\varepsilon)+F[\boldsymbol{V}(\varepsilon)]}{k T}\right] \\
= & \int d \varepsilon^{\frac{D(D+1)}{2}} \exp \left[-\frac{G[\boldsymbol{V}(\varepsilon)]}{k T}\right] \\
= & \exp \left[-\frac{G[\boldsymbol{V}(\mathbf{0})]}{k T}\right] \int d \varepsilon^{\frac{D(D+1)}{2}} \\
& \times \exp \left[-\frac{V_{p}}{2 k T} \sum_{i j k l}^{D} B_{i j k l} \varepsilon_{i j} \varepsilon_{k l}\right],
\end{aligned}
$$

where the last equality is correct for stable reference states (described by positive difinite quadratic form (4)) when the system is large enough, i.e. when the strain fluctuations are small.

The following relation comes from (15) and (7):

$$
\left\langle\varepsilon_{i j} \varepsilon_{k l}\right\rangle=\frac{k T S_{i j k l}}{V_{p}}
$$

where

$$
\langle f\rangle \equiv \frac{\int d \varepsilon^{\frac{D(D+1)}{2}} f \exp (-G[\boldsymbol{V}(\varepsilon)] / k T)}{\int d \varepsilon^{\frac{D(D+1)}{2}} \exp (-G[\boldsymbol{V}(\varepsilon)] / k T)}
$$

denotes the thermodynamic averaging of a function $f$ with the partition function (14). In particular, the average (equilibrium) volume at the pressure $p$ is calculated as

$$
V_{p}=\langle|\operatorname{det}(\boldsymbol{h})|\rangle
$$

It is worth to add that the compressibility, $K_{T}=-\frac{1}{V_{p}}\left(\frac{\partial V}{\partial p}\right)_{T}$, can be obtained from the equation:

$$
\begin{aligned}
\left\langle(\Delta V)^{2}\right\rangle & \equiv \frac{\int d \varepsilon^{\frac{D(D+1)}{2}}(\Delta V)^{2} \exp (-G[\boldsymbol{V}(\varepsilon)] / k T)}{\int d \varepsilon^{\frac{D(D+1)}{2}} \exp (-G[\boldsymbol{V}(\varepsilon)] / k T)} \\
& =k T V_{p} K_{T} .
\end{aligned}
$$

\section{Determination of the thermodynamic averages}

The strain tensor is defined with respect to the reference state which corresponds to the equilibrium state of the system at the given pressure. Since, in general, the equilibrium state is not known in advance (i.e. before the simulation), one might think that to calculate the thermodynamic averages of the form (17) which depend on the strain components, it is necessary to perform an initial run to determine the reference state. (Moreover, as the reference state must be determined very accurately, the initial run would not be computationally cheap.) The present, constant pressure 
method does not require, however, any extra initial run to determine the reference state. This is illustrated below for a two-dimensional system. (The three-dimensional case is discussed in detail elsewhere [117].)

The integrals over the strain components can be converted into integrals over independent components of the box matrix. Using the formula (11) and denoting the Jacobian of the transformation $\left(h_{x x}, h_{y y}, h_{x y}\right) \rightarrow\left(\varepsilon_{x x}, \varepsilon_{y y}, \varepsilon_{x y}\right)$ by $j(\boldsymbol{h}, \boldsymbol{H})$ one can express the average (with respect to $\left.\varepsilon\right)$ in Eq.(17) by the ratio of the averages with respect to $\boldsymbol{h}$ :

$$
\langle f\rangle=\langle f j(\boldsymbol{h}, \boldsymbol{H})\rangle_{h} /\langle j(\boldsymbol{h}, \boldsymbol{H})\rangle_{h},
$$

where, for $f \equiv f\left(\boldsymbol{s}^{(N)}, \boldsymbol{\Omega}^{(N)}, \boldsymbol{\varepsilon}(\boldsymbol{h}, \boldsymbol{H})\right)$ being any (smooth) function of the configuration of a two-dimensional system, the averages $\langle\ldots\rangle_{h}$ are defined as

$$
\langle f\rangle_{h} \equiv \frac{\int d h_{x x} \int d h_{y y} \int d h_{x y} V^{N} \int d \boldsymbol{s}^{(N)} \int d \boldsymbol{\Omega}^{(N)} f\left(\boldsymbol{s}^{(N)}, \boldsymbol{\Omega}^{(N)}, \boldsymbol{h}, \boldsymbol{H}\right) \exp [-(U+p V) / k T]}{\int d h_{x x} \int d h_{y y} \int d h_{x y} V^{N} \int d \boldsymbol{s}^{(N)} \int d \boldsymbol{\Omega}^{(N)} \exp [-(U+p V) / k T]} .
$$

To eliminate the strain from the boundary dependences of the integrals in (21), the 'normalized' (scaled to a unit square) positions of the particles, $\boldsymbol{s}_{i}=\boldsymbol{h}^{\mathbf{- 1}} \cdot \boldsymbol{r}_{i}(i=1, \ldots, N)$ are used; the box matrix $\boldsymbol{h}$ is symmetric. For the symmetric box matrix in two dimensions the Jacobian

$$
\begin{aligned}
j(\boldsymbol{h}, \boldsymbol{H}) & \equiv \frac{\partial\left(\varepsilon_{x x}, \varepsilon_{y y}, \varepsilon_{x y}\right)}{\partial\left(h_{x x}, h_{y y}, h_{x y}\right)} \\
& =\frac{\left(h_{x x}+h_{y y}\right)\left(h_{x y}^{2}-h_{x x} h_{y y}\right)}{2\left(H_{x y}^{2}-H_{x x} H_{y y}\right)^{3}} \\
& =j_{h}(\boldsymbol{h}) j_{H}(\boldsymbol{H})
\end{aligned}
$$

is a product of a function which depends only on the components of the box matrix, $\boldsymbol{h}$, and a function which depends only on the components of the reference box matrix, $\boldsymbol{H}$, which can be thought of as (unknown) constants. Thus, the thermodynamic average of the function $f$ can be written as

$$
\begin{aligned}
\langle f\rangle & =\left\langle f j_{h}\right\rangle_{h} /\left\langle j_{h}\right\rangle_{h} \\
& =\frac{\int d h_{x x} \int d h_{y y} \int d h_{x y} \int d \boldsymbol{s}^{(N)} \int d \boldsymbol{\Omega}^{(N)} f \exp \left(-G_{m i c r o} / k T\right)}{\int d h_{x x} \int d h_{y y} \int d h_{x y} \int d \boldsymbol{s}^{(N)} \int d \boldsymbol{\Omega}^{(N)} \exp \left(-G_{m i c r o} / k T\right)}
\end{aligned}
$$

where the 'microscopic free enthalpy'

$$
G_{m i c r o}=k T[(N+1) \ln V+\ln \operatorname{Tr}(\boldsymbol{h})]-U+p V
$$

does not depend on the reference system.

The equation (23) states that at isotropic pressure the Monte Carlo simulation can be performed without knowing the structural parameters (i.e. the reference state) of the system at equilibrium in advance (i.e. before performing the simulation run in which the elastic properties of the system are 
computed). This is in contrast to the general case of an anisotropic stress when the microscopic enthalpy is an explicit function of the strain and when one has to know the reference state before the simulations.

The simplest scheme to determine $S_{i j k l}$ in (16) is to store during the simulations the values of the box matrix components $h_{i j}$ in a file. After finishing the run, the stored data can be used to compute the average (equilibrium) values of the components of the box matrix, $\left\langle h_{i j}\right\rangle=\left(h_{r e f}\right)_{i j} \equiv H_{i j}$, by substituting $f=h_{i j}$ in (23). The calculated equilibrium (reference) values can then be used to compute the strain tensor components, $\varepsilon_{i j}$, what, in turn, makes possible calculation of the elastic compliances according to (20).

Remark: It is worth to add that the method used in the present work does not require creating any file in which the data are stored. Namely, it follows from (11), (16) and (18) that to obtain the compliances it is enough to average during the simulations a few monomials consisted of the box matrix components of powers not exceeding four [117]. The average values of these monomials (including the average values of the box matrix components which define the reference state) can be substituted to the formula (16) at the end of the run what gives the elastic compliances. However, creation of a file with the 'history' of the box evolution not only offers a simple and convenient scheme for determination of the compliances, $S_{i j k l}$, but also helps to estimate the equilibration 'time' and the statistical errors of the results obtained.

\section{Details of the simulations}

In the $\mathrm{MC}$ simulations discussed in this paper two kinds of trial moves were performed. The first concerned (simultaneous) changes of the molecular mass centre position and the molecular orientation; the acceptance ratio was kept close to 30 percent. The maximal amplitudes of the allowed changes of the translational degrees of freedom and the rotational one (in radians) were the same. (Other choices, e.g. based on equality of average kinetic energies of translational and rotational degrees of freedom [110] give the same results, within the experimental error.) The second kind of moves, tried about $N^{1 / 2}$ times less frequently, concerned changes of the components of the symmetric box matrix only; the orientations, $\boldsymbol{\Omega}_{i}$, and the scaled positions, $\boldsymbol{s}_{i}$, of the particles were not changed. These moves determined the size and the shape of the box, and their acceptance ratio was close to 20 percent.

It is well known that the anisotropy of the periodic box can modify (even qualitatively, see e.g. $[118,119])$ the thermodynamic properties of the studied system. To minimize the influence of the box anisotropy, samples of shapes close to the square have been used. The simulations of the pentamers and the $\mathrm{A}$ and $\mathrm{B}$ structures of the heptamers were carried out for samples being consecutive quadruplings of a minimum sample, which consisted of 8 horizontal rows parallel to 
the $x$-axis, each row composed of 7 molecules. This determined the sizes of the studied systems: $N=56,224,896$. As the $\mathrm{C}$ structure of the heptamers cannot be arranged in a $8 \times 7$ periodic lattice, only samples of $N=224,896$ heptamers were simulated in this case. Additionally, simulations of $N=36,144,576$ were performed for the C structure. In this case the minimum sample consisted of 6 horizontal rows parallel to the $x$-axis, each row composed of 6 molecules. Typical lengths of the runs were equal $3 \times 10^{6}$ trial steps per particle (cycles) for $N=36,5 \times 10^{6}$ cycles for $N=56,144$, $10^{7}$ cycles for $N=224$, and $2 \times 10^{7}$ for $N=576,896$. Some longer runs were also performed to compare different random number generators and to check the convergence of the method. Up to 10 percent of the typical simulation length was considered as the 'equilibration time' and not taken into account in the averaging procedures. This can be thought of as using large 'safety' margin because the analysis of the evolution of the box matrix components as well as the particle positions and orientations distributions indicated that the equilibrium was reached for 'times' by one order of magnitude smaller.

\section{HIGH DENSITY STRUCTURES OF THE PENTAMERS AND THE HEPTAMERS}

Various structures of the pentamers and heptamers have been found in computer simulations [5358]. The simulations proved that in both cases there exist the fluid phase and some solid phases which represent various orientational orderings, e.g. (almost) free rotation, (strongly) hindered rotation, and frozen rotation [54,56-58]. The latter ordering was observed at the highest densities which are the subject of the present work; at these densities the molecules in both systems only vibrate around their lattice positions and librate around their preferred orientations.

In the system of the pentamers only one structure without molecular rotation has been found [53, $54,56]$. This structure contains two pentamers in its rectangular unit cell and its geometry at close packing can be seen in Fig.2a; see also Table I. The 'row structure' shown in Fig.2a consists of rows of closely packed pentamers. The rows are parallel to the $x$-axis, and the molecules in the consecutive rows are anti-parallel to each other.

In contrast to pentamers, the heptamers form (at least) three different (thermodynamically stable or metastable) structures without molecular rotation at the highest densities [55, 58]. All these structures, shown in Fig.2b-d, are of rectangular unit cells. The structures in Fig.2bc are similar to the structure formed by the pentamers, and they differ from each other by the relative positions of the rows. One of them, denoted by B, was obtained by a slow compression of the system from the lower density, orientationally disordered, phase of triangular lattice. The close packed version of this structure can be seen in Fig.2b. It is interesting to notice that the structure $\mathrm{B}$ is not the most dense structure which can be obtained by packing rows of the closely packed

heptamers of the same orientation within a row, see Table I. In Fig.2c one can see a structure, further referred to as A, which is the most dense of such row-structures [58]. The structures A and 
B have two heptamers in the unit cell. Obviously, at densities high enough (i.e. when the pressure, $p$, tends to infinity), the structure A must be more stable than B. The structure A is, however, not the most dense structure of the heptamers. Even more dense structure of the heptamers, further referred to as the $\mathrm{C}$ structure, is shown in Fig.2d. The structure $\mathrm{C}$ is the most dense structure of the heptamers we know and its unit cell contains four molecules which are not oriented along the horizontal molecular 'rows', see Table I. Obviously, in the limit of $p \rightarrow \infty$ this structure must be more stable than the structure A.

It is worth to notice that none of the latter two structures (A and $\mathrm{C}$ ) was obtained by either a spontaneous transition from the structure B or by compressing any other phase. This is despite that (i) at close packing both these structures are denser than B and (as it can be seen in Fig.3) (ii) their izotherms are located below the isotherm corresponding to the latter one in the whole density range where they are mechanically stable. This indicates that the local minima of the free energy corresponding to the solid structures of heptamers are very deep. In consequence, the time necessary for occurrence of any structural transition in the heptamer system at high densities is much longer than the times of the simulations performed for the studied sizes of the samples. This allows one for determination of the elastic properties of the studied structures for a whole range of high pressures despite that at a randomly chosen pressure in this range we know with probability equal to unity that only one of these structures can be thermodynamically stable.

The unit cell parameters of the studied solids at close packing (i.e. in the limit of infinite pressure) are collected in Table I. The corresponding parameters at a few finite pressures are shown in Table II.

\section{ELASTIC PROPERTIES}

As it was mentioned in the introduction, no defects were present in the structures studied in this work. Defects are, however, unavoidable at densities below the close packing in the thermodynamic limit. Thus, in this context, as well as in the context of the well known problem concerning the lack of any true translational ordering in two dimensions [59, 60], the obtained elastic constants and compliances can be thought of as describing local elastic properties of the studied systems.

\section{A. Simulation results}

In Tables III-VI the dimensionless elastic compliances, $S_{i j k l}^{*} \equiv S_{i j k l} \sigma^{2} / k T$, obtained for the pentamers and the heptamers are collected. (We do not show the compliances, $S_{x x x y}^{*}, S_{x y y y}^{*}$ which have to be zero by the symmetry requirements.) It can be seen that the elastic compliances obtained for systems as small as $N=56$ particles differ by only a few percent from the estimates obtained for the thermodynamic limit $N \rightarrow \infty$. The same is true for the Poisson ratios which can be obtained 
from the compliances by (10).

It is worth to notice that the $S_{x x y y}^{*}$ compliances are negative for the rectangular structure of the pentamers and the B structure of the heptamers. Such a behavior is typical for materials existing in nature and it implies that the Poisson ratios of these structures are positive. In contrast to them, the remaining (more dense) structures of the heptamers exhibit positive compliances $S_{x x y y}^{*}$, i.e. negative Poisson ratios. Thus, the $\mathrm{A}$ and $\mathrm{C}$ structures are very simple examples of anisotropic auxetic phases. The possibility that some non-convex molecules may form auxetic phases has been pointed out by one of the authors in the past $[69,70]$.

Part of the results presented in tables II-VI was obtained by using a few HP workstations. It appears that the simulations on these workstations can be speed up substantially (almost twice) when the (pseudo)random numbers are generated by using the SNWS generator proposed by Holian et al [120] which is based on the Weyl sequence. This generator, which is also convenient to use for parallel computations, has been already tested by us in the case of free energy computations for the f.c.c. and h.c.p. crystals of hard spheres [121]. Most of the runs in the present simulations of the elastic constants has been done by using the SNWS Holian's generator and its simple modifications [122]. In Fig.4, representative examples of the accumulation plots are shown for the elastic compliances and the $x y$ and $y x$ Poisson ratios calculated for the rectangular structure of the pentamers by using two random number generators, DRAND48 and the SNWS Holian's generator. It can be seen that the results obtained for both the generators agree within the experimental error. This encourages one to use the Holian's SNWS generator in other Monte Carlo simulations.

Looking at the Fig.4 one can also estimate the convergence of the computed data. As expected $[45,108]$ the convergence of the simulation results in the case of the elastic properties is much slower than, e.g., in the case of the equation of state. The slow convergence of the fluctuation method is also illustrated in Fig. 5 where one can see the accumulation plots of the elastic properties obtained for $N=896$ pentamers for a run consisted of $4.2 \times 10^{7} \mathrm{MC}$ cycles.

It is well known that the real time of simulations using any computational method can be reduced if the method can be parallelized. Since the present method does not require to know the reference state prior to simulations it can be fully parallelized and easily implemented on a parallel computer. In Table VII we collected the results obtained for the elastic constants for three runs of the same length $4 \times 10^{7} \mathrm{MC}$ cycles (after equilibration). The first run (a) was performed by a single processor starting from a perfectly ordered rectangular structure; the initial $5 \times 10^{5}$ cycles were spent for equilibration. The other two runs were performed parallelly by 16 processors. In the case (b) the starting configurations corresponded to the configurations obtained within the first run after $(4+m) \times 10^{5}$ MC cycles where $m=1, \ldots, 16$ is the processor number; the sequences of random numbers were different in each case. No equilibration cycles were performed in this case. In the case (c) all the initial configurations were the same as the initial configuration in the case (a) but the sequences of the random numbers were different; $5 \times 10^{5}$ equilibration cycles were performed in 
each of these runs. It can be seen that the obtained results are in agreement within the combined statistical errors.

\section{B. Free volume approximation}

Some properties of the hard-core systems, like pressure or elastic constants, are divergent in the close-packing limit. Other quantities, like compliances, tend to zero in this limit. In general, to analyze numerically quantities which diverge or tend to zero, it is meaningful to rescale them by their asymptotics obtained from a theoretical research. In the particular case of the hard-core systems the asymptotics are offered by the free volume approximation [15, 26, 123-129].

The essence of the free volume approach consists in the observation that in the condensed matter phases the molecules can be thought of as moving in shells formed by their nearest neighbors. This approximation can be proven to give asymptotically exact results for the equation of state of hard spheres (or hard discs) in the close packing limit [124]. It is well known that at high densities the equation of state of some other hard-body systems is also well approximated by the free volume theory $[23,128,130]$. The free volume predicts also correctly the asymptotic form of the elastic constants of hard spheres and discs $[35,38,45,46]$. It will be shown below that the free volume approximation works also well in the case of the studied phases of pentamers and heptamers.

It has been shown in [124] that for the $D$-dimensional hard spheres of the diameter $\sigma$ the free volume, $v^{(F V)}$, near the close packing is proportional to the $g$-th power ( $g$ being the number of the molecular degrees of freedom, i.e. $g=2$ for hard discs and $g=3$ for hard spheres) of the excess distance, $\delta=a-\sigma$, between the centers of the neighboring spheres at their lattice site positions (which are distanced by $a$ ) [124]

$$
v^{(F V)}=c(\delta)^{g}
$$

where

$$
\delta / \sigma \equiv \delta^{*}=\left(V / V_{c p}\right)^{1 / D}-1
$$

$V$ is the volume of the system, $V_{c p}$ is the volume of the considered structure at close packing, and

$c$ depends on the shape of the free volume. Applying this formula to anisotropic hard bodies and assuming that near close packing $c$ does not depend on the volume per particle (what means that the free volume does not change its shape when the volume of the system is changed by isotropic pressure) one can neglect the constant $c$ and write the configurational free energy per one 'molecule' (body) in the form:

$$
f^{(F V)}=-k T \ln v^{(F V)}=-g k T \ln \left(\left(V / V_{c p}\right)^{1 / D}-1\right) .
$$


This leads to the following equations for the pressure and the bulk modulus:

$$
\begin{aligned}
p^{(F V)} & =-\frac{\partial f}{\partial(V / N)}=\frac{g N k T}{D V}\left(1+\frac{1}{\delta^{*}}\right) \\
B_{T}^{(F V)} & =-V \frac{\partial p}{\partial V}=1 / K_{T}^{(F V)}= \\
& =\frac{g N k T}{D V}\left(1+\frac{1+\frac{1}{D}}{\delta^{*}}+\frac{\frac{1}{D}}{\left(\delta^{*}\right)^{2}}\right),
\end{aligned}
$$

where $\delta^{*}$ is defined in (26).

As it is easy to see, the pressure shows singularity of the form $\left(\delta^{*}\right)^{-1}$ whereas the leading singularity of the bulk modulus is of the form $\left(\delta^{*}\right)^{-2}$. The elastic constants are expected to show the same (leading) singularity as the bulk modulus [35].

In the Fig. 6 we show that the free volume approximation works well both in the case of pentamers and heptamers for which $D=2$ and $g=3$. It can be seen there that the ratios of the elastic constants to the bulk modulus obtained from the free volume approximation are well approximated by linear functions of the inverse density. In particular, as predicted by the free volume theory, the

ratios $p / p^{(F V)}$ and $B_{T} / B_{T}^{(F V)}$ tend to unity at close packing. In the first case the accuracy of the experimental determination of this limit is much higher (close to one promile) than in the second case (about two percent).

The ordinates of the (linear) fits shown in Fig.6 at volumes corresponding to close packings of the studied structures characterize quantitatively the singular behavior in the vicinity of close packings of studied structures. The obtained coefficients and directional Poisson ratios are collected in Table VIII. It can be seen that some of the values of directional Poisson ratios are negative.

\section{SUMMARY AND CONCLUSIONS}

Elastic properties of dense solid structures for pentamers and heptamers were determined by analysis of the box matrix evolution in Monte Carlo simulations. It has been shown that simulations of systems as small as $N=56$ give results which differ by only a few percent from the results obtained by extrapolation to the thermodynamic limit.

The asymptotics of the obtained equation of state, bulk modulus, and elastic constants are in agreement with predictions of a simple version of the free volume theory. The coefficients of the leading singularities of pressure and elastic constants in the vicinity of the close packing have been determined. These coefficients can be useful to construct and to test various theoretical approximations concerning elasticity of molecular systems.

We should note that the computations performed show that the Poisson ratios of the most dense structures of heptamers are negative. This result confirms earlier suggestions that certain 
non-convex molecules may form phases of anomalous Poisson ratio [70]. We should stress that the heptamers are not an exception in this aspect; analytical and computer simulation results concerning other molecules forming thermodynamically stable auxetic phases will be discussed elsewhere.

It is worth to add that the simulation method applied in this paper does not require using the structural parameters (reference state) of the unit cell of the studied system as an simulation input. The reference (equilibrium) state is determined within the same run in which the elastic properties are determined. This saves a substantial amount of the simulation time. This also allows one to increase the accuracy of the results by extending the length of the simulations simply by continuing them from the place in which they were stopped.

Although the applied method of elastic properties determination does not converge as quickly as methods using other thermodynamic ensembles (e.g. the NVT ensemble), its simplicity (there is no need to apply microscopic expressions for the pressure or its derivatives) and possibility to calculate all the elastic constants in a single run encourages one for applying it in various systems. In particular, this method seems to be preferable in situations where one needs easy and quick estimates of the elastic properties for systems with complex (e.g. molecular) interactions for which calculations of the forces are either time-consuming or nontrivial. This is, e.g., the case of some systems forming isotropic auxetic phases which are the subject of separate works.

\section{Acknowledgments}

We are grateful to Dr. B. L. Holian for sending to us the code of the SNWS (pseudo)random number generator at the very beginning stage of this project. Part of this work was supported by the grant 4T11F 01023 of the Polish Committee for Scientific Research (KBN). Part of the calculations was performed at the Poznań Computer and Networking Center (PCSS).

\section{References}

[1] L. Onsager, Ann. NY Acad. Sci. 51, 3441 (1949).

[2] B. J. Adler and T. E. Wainwright, Phys. Rev. 127, 359 (1962).

[3] B. J. Adler, W. G. Hoover, and T. E. Wainwright, Phys. Rev. Lett. 11, 241 (1963).

[4] D. J. Bernal, Proceedings of Royal Society of London A 280, 299 (1963).

[5] W. G. Hoover and F. H. Ree, J. Chem. Phys. 49, 3609 (1968).

[6] S. Cargill, J. Applied Physics 43, 2727 (1972).

[7] J. Vieillard-Baron, J. Chem. Phys. 56, 4729 (1972).

[8] P. Pierański, J. Małecki, W. Kuczyński, and K. W. Wojciechowski, Philosophical Magazine A 37, 107 (1980). 
[9] P. Pierański, J. Małecki, and K. W. Wojciechowski, Molecular Physics 40, 225 (1981).

[10] A. C. Brańka, P. Pierański, and K. W. Wojciechowski, J. Phys. Chemistry of Solids 43, 817 (1983).

[11] A. Bonissent, P. Pierański, and P. Pierański, Philosophical Magazine A 50, 57 (1984).

[12] D. Frenkel and B. M. Mulder, Molec. Phys. 55, 1171 (1985).

[13] A. Stroobants, H. N. W. Lekkerkerker, and D. Frenkel, Phys. Rev. Lett. 57, 1452 (1986).

[14] M. S. Wertheim, J. Chem. Phys. 87, 7323 (1987), see also references therein.

[15] K. W. Wojciechowski, Phys. Lett. A 122, 377 (1987).

[16] T. Boublik, Molec. Phys. 68, 191 (1989), see also references therein.

[17] K. W. Wojciechowski, D. Frenkel, and A. C. Brańka, Phys. Rev. Lett. 66, 3168 (1991).

[18] D. Frenkel, in Liquids, Freezing and the Glass Transition, edited by J. P. Hansen, D. Levesque, and J. Zinn-Justin (Elsevier, 1991).

[19] K. W. Wojciechowski, J. Chem. Phys. 94, 4099 (1991).

[20] G. T. Evans, Molec. Phys. 77, 969 (1992).

[21] J. A. C. Veerman and D. Frenkel, Phys. Rev. A 45, 5632 (1992).

[22] C. Vega, E. A. P. Paras, and P. A. Monson, J. Chem. Phys. 96, 9060 (1992).

[23] K. W. Wojciechowski, Phys. Rev. B 46, 26 (1992).

[24] M. P. Allen, G. T. Evans, D. Frenkel, and B. M. Mulder, Advanced Chemical Physics LXXXVI, 1 (1993), and references therein.

[25] A. P. Malanoski and P. A. Monson, J. Chem. Phys. 107, 6899 (1997).

[26] M. Schmidt and H. Löwen, Phys. Rev. E 55, 7228 (1997).

[27] R. Blaak and B. M. Mulder, Phys. Rev. E 58, 5873 (1998).

[28] P. I. C. Teixeira, A. J. Masters, and B. M. Mulder, Molec. Crys. Liquid Crys. 323, 167 (1998).

[29] K. W. Wojciechowski, in Statistical Physics: Experiments, Theories, and Computer Simulations, edited by M. Tokuyama and I. Oppenheim (World Scientific, Singapore, 1998), p. 69.

[30] R. Bruinsma, Phys. Rev. E 63, 061705 (2001).

[31] C. Vega and L. C. MacDowell, J. Chem. Phys. 114, 10411 (2001).

[32] M. A. Bates, M. G. Noro, and D. Frenkel, J. Chem. Phys. 116, 7217 (2002).

[33] C. McBride and C. Vega, J. Chem. Phys. 116, 1757 (2002).

[34] J. Dzubiella, C. N. Likos, and H. Löwen, J. Chem. Phys. 116, 9518 (2002).

[35] F. H. Stillinger and Z. W. Salsburg, J. Chem. Phys. 46, 3962 (1967).

[36] M. V. Jaric and U. Mohanty, Phys. Rev. Lett. 58, 230 (1987).

[37] E. Velasco and P. Tarazona, Phys. Rev. A 36, 979 (1987).

[38] D. Frenkel and A. J. C. Ladd, Phys. Rev. Lett. 59, 1169 (1987).

[39] K. J. Runge and G. V. Chester, Phys. Rev. A 36, 4852 (1987).

[40] H. Xu and M. Baus, Phys. Rev. A 38, 4348 (1988).

[41] H. Löwen, J. Phys.: Cond. Matter 2, 8477 (1990).

[42] B. B. Laird, J. Chem. Phys. 97, 2699 (1992). 
[43] O. Farago and Y. Kantor, Phys. Rev. E 61, 2478 (2000).

[44] K. V. Tretiakov and K. W. Wojciechowski, unpublished.

[45] K. W. Wojciechowski and A. C. Brańka, Phys. Lett. A 134, 314 (1989).

[46] K. W. Wojciechowski, Molec. Phys. 72, 955 (1991).

[47] S. Sengupta, P. Nielaba, M. Rao, and K. Binder, Phys. Rev. E 61, 1072 (2000).

[48] M. A. Bates and D. Frenkel, Phys. Rev. E 61, 5223 (2000).

[49] S. Sengupta, P. Nielaba, and K. Binder, Phys. Rev. E 61, 6294 (2000).

[50] K. W. Wojciechowski, K. V. Tretiakov, A. C. Brańka, and M. Kowalik, unpublished.

[51] A. A. Berlin, L. Rothenburg, and R. J. Bathurst, Polymer-Plastics Technology and Engineering 35, 605 (1996).

[52] A. C. Brańka and K. W. Wojciechowski, Phys. Lett. A 101, 349 (1984).

[53] A. C. Brańka and K. W. Wojciechowski, Molec. Phys. 72, 941 (1991).

[54] A. C. Brańka and K. W. Wojciechowski, Molec. Phys. 78, 1513 (1993).

[55] K. V. Tretiakov, Ph.D. thesis, Institute of Molecular Physics Polish Academy of Sciences (2000).

[56] K. V. Tretiakov and K. W. Wojciechowski, TASK Quarterly 5, 331 (2001).

[57] K. V. Tretiakov and K. W. Wojciechowski, J. Phys.: Cond. Matter 14, 1261 (2002).

[58] K. Wojciechowski and K. V. Tretiakov, Computational Methods in Science and Technology 6, 101 (2000).

[59] S. K. Shina, in Ordering in two dimensions (North Holland, New York, 1980), see also references therein.

[60] K. Strandburg, Rev. Mod. Phys. 60, 161 (1988), see also references therein.

[61] L. D. Landau, E. M. Lifszyc, A. M. Kosevich, and I. P. Pitaevskij, Theory of Elasticity (Pergamon Press, London, 1986).

[62] R. F. Almgren, J. Elasticity 15, 427 (1985).

[63] K. W. Wojciechowski, Molec. Phys. 61, 1247 (1987).

[64] R. Lakes, Science 235, 1038 (1987).

[65] E. A. Friis, R. S. Lakes, and J. Park, J. Material Science 23, 4406 (1988).

[66] R. J. Bathrust and L. Rothenburg, Int. J. Eng. Sci. 26, 373 (1988).

[67] A. W. Lipsett and A. I. Beltzer, Journal of the Acoustical Society of America 84, 2179 (1988).

[68] K. E. Evans, J. Phys. D 22, 1870 (1989).

[69] K. W. Wojciechowski, Phys. Lett. A 60, 1989 (1989).

[70] K. W. Wojciechowski and A. C. Brańka, Phys. Rev. A 40, 7222 (1989).

[71] S. Hirotsu, Macromolecules 23, 903 (1990).

[72] S. Hirotsu, J. Chem. Phys. 94, 3949 (1991).

[73] K. E. Evans, Endeavour, New Series 15, 170 (1991).

[74] K. E. Evans, I. J. Hutchinson, and S. C. Rogers, Nature 353, 124 (1991).

[75] L. Rothenburg, A. A. Berlin, and J. Bathurst, Nature 354, 470 (1991). 
[76] G. Wei and S. F. Edwards, Computational Polymer Science 2, 44 (1992).

[77] G. Wei, J. Chem. Phys. 96, 3226 (1992).

[78] K. E. Evans, M. A. Nkansah, and I. J. Hutchinson, Acta Metall. Mat. 40, 2463 (1992).

[79] G. W. Milton, J. Mech. Phys. Solids 40, 1105 (1992).

[80] R. Lakes, Advanced Materials 5, 293 (1993).

[81] D. H. Boal, U. Seifert, and J. C. Shillcock, Phys. Rev. E 48, 4274 (1993).

[82] K. W. Wojciechowski, Molecular Physics Reports 10, 129 (1995).

[83] E. O. Martz, R. S. Lakes, and J. B. Park, Cellular Polymers 15, 349 (1996).

[84] K. W. Wojciechowski and K. V. Tretiakov, Computational Methods in Science and Technology 1, 25 (1996).

[85] M. A. Loureiro and R. S. Lakes, Cellular Polymers 16, 349 (1997).

[86] D. Prall and R. Lakes, Int. J. Mech. Sci. 39, 305 (1997).

[87] U. D. Larsen, O. Sigmund, and S. Bouwstra, J. Material Science 6, 99 (1997).

[88] P. S. Theocaris, G. E. Stavroulakis, and P. D. Panagiotopoulos, Archive of Applied Mechanics 67, 274 (1997).

[89] R. H. Baughman, J. M. Shacklette, A. A. Zakhidov, and S. Stafstrom, Nature 392, 362 (1998).

[90] K. W. Wojciechowski, in Statistical Physics: Experiments, Theories, and Computer Simulations, edited by M. Tokuyama and I. Oppenheim (World Scientific, Singapore, 1998), p. 107.

[91] C. B. He, P. W. Liu, and A. C. Griffin, Macromolecules 31, 3145 (1998).

[92] G. Wei and S. F. Edwards, Phys. Rev. E 58, 6173 (1998).

[93] V. V. Novikov and K. W. Wojciechowski, Physics of the Solid State 41, 1970 (1999).

[94] R. H. Baughman, S. O. Dantas, S. Stafstrom, A. A. Zakhidov, T. B. Mitchell, and D. H. E. Dubin, Science 288, 2018 (2000).

[95] K. E. Evans and A. Alderson, Advanced Materials 12, 617 (2000).

[96] M. Bowick, A. Cacciuto, G. Thorleifsson, and A. Travesset, Phys. Rev. Lett. 87, 148103 (2001).

[97] B. Brandel and R. S. Lakes, J. Material Science 36, 5885 (2001).

[98] K. W. Wojciechowski and V. V. Novikov, TASK Quarterly 5, 5 (2001).

[99] A. A. Vasiliev, S. V. Dmitriev, Y. Ishibashi, and T. Shigenari, Phys. Rev. B 65, 94101 (2002).

[100] M. Parrinello and A. Rahman, J. Chem. Phys. 76, 2662 (1982).

[101] J. R. Ray and A. Rahman, J. Chem. Phys. 80, 4423 (1984).

[102] J. R. Ray and A. Rahman, J. Chem. Phys. 82, 4243 (1985).

[103] K. W. Wojciechowski and K. V. Tretiakov, Computational Physics Communications 121-122, 528 (1999).

[104] D. R. Squire, A. C. Holt, and W. G. Hoover, Physica (Amsterdam) 42, 388 (1968).

[105] M. Sprik, R. W. Impey, and M. L. Klein, Phys. Rev. B 29, 4368 (1984).

[106] J. R. Ray, M. C. Moody, and A. Rahman, Phys. Rev. B 32, 733 (1985).

[107] J. R. Ray, M. C. Moody, and A. Rahman, Phys. Rev. B 33, 895 (1986). 
[108] T. Çagin and J. R. Ray, Phys. Rev. B 38, 7940 (1988).

[109] Z. Zhou, J. Chem. Phys. 114, 8769 (2001).

[110] K. W. Wojciechowski, A. C. Brańka, and M. Parinello, Molec. Phys. 53, 1541 (1984).

[111] D. Frenkel and A. J. C. Ladd, J. Chem. Phys. 18, 3188 (1984).

[112] D. C. Wallace, Thermodynamics of Crystals (Wiley, New York, 1972).

[113] J. H. Weiner, Statistical Mechanics of Elasticity (Wiley, New York, 1983).

[114] M. Parrinello and A. Rahman, Phys. Rev. Lett. 45, 1196 (1980).

[115] M. Parrinello and A. Rahman, J. Applied Physics 52, 7182 (1981).

[116] S. Nosé and M. L. Klein, Molec. Phys. 50, 1055 (1983).

[117] K. W. Wojciechowski, unpublished.

[118] K. W. Wojciechowski, P. Pierański, and J. Małecki, J. Chem. Phys. 76, 6710 (1982).

[119] K. W. Wojciechowski, P. Pierański, and J. Małecki, J. Phys. A 16, 2197 (1983).

[120] B. L. Holian, O. E. Percus, T. T. Warnock, and P. A. Whitlock, Phys. Rev. E 50, 1607 (1994).

[121] K. V. Tretiakov and K. W. Wojciechowski, Phys. Rev. E 60, 7626 (1999).

[122] K. W. Wojciechowski, Computational Methods in Science and Technology 5, 81 (1999).

[123] J. A. Barker, Lattice Theories of the Liquid State (Pergamon, Oxford, 1963).

[124] F. H. Stillinger, E. A. DiMarzio, and R. L. Kornegay, J. Chem. Phys. 40, 1564 (1964).

[125] W. G. Hoover, W. T. Ashurst, and R. Grover, J. Chem. Phys. 57, 1259 (1972).

[126] W. G. Hoover, N. E. Hoover, and K. Henson, J. Chem. Phys. 70, 1837 (1979).

[127] K. W. Wojciechowski and A. C. Brańka, J. Phys. Chemistry of Solids 45, 913 (1984).

[128] M. P. Taylor, R. Hentschke, and J. Herzfeld, Phys. Rev. Lett. 62, 800 (1989).

[129] K. W. Wojciechowski, Physica A 232, 723 (1996).

[130] K. W. Wojciechowski, A. C. Brańka, and D. Frenkel, Physica A 196, 519 (1993). 
TABLE I: Parameters of the studied structures at close packing, see Fig.2; the distances are given in units of $\sigma$. The molecule 0 is always placed at the origin of the coordinate system, i.e. for each structure $x_{0}=y_{0}=0$. The two-dimensional volume (i.e. the area) per particle of the considered structure at close packing is equal to $a_{c p}^{(\text {structure })} b_{c p}^{\text {(structure) }} / n_{u}$, where $n_{u}=2$ for the structure $=P, A, B$ and $n_{u}=4$ for the structure $=C$.

\begin{tabular}{|c|c|c|c|c|c|}
\hline \multicolumn{6}{|c|}{ Pentamers P } \\
\hline & $=2.4048671732$ & \multicolumn{3}{|c|}{$b_{c p}^{(P)}=4.2360679772$} & $\phi_{0}=0$ \\
\hline$x_{1}$ & $=1.0131106571$ & \multicolumn{3}{|c|}{$y_{1}=b_{c p}^{(P)} / 2$} & $\phi_{1}=\pi / 5$ \\
\hline \multicolumn{6}{|c|}{ Heptamers A } \\
\hline$a_{c p}^{(A)}$ & $=3.0566685376$ & \multicolumn{3}{|c|}{$b_{c p}^{(A)}=5.5150210832$} & $\phi_{0}=0$ \\
\hline$x_{1}$ & $=1.9144263193$ & \multicolumn{3}{|c|}{$y_{1}=b_{c p}^{(A)} / 2$} & $\phi_{1}=\pi / 7$ \\
\hline \multicolumn{6}{|c|}{ Heptamers B } \\
\hline$a_{c p}^{(B)}$ & $=3.0566685376$ & \multicolumn{3}{|c|}{$b_{c p}^{(B)}=5.5382990167$} & $\phi_{0}=0$ \\
\hline & $=1.3656999121$ & \multicolumn{3}{|c|}{$y_{1}=b_{c p}^{(B)} / 2$} & $\phi_{1}=\pi / 7$ \\
\hline \multicolumn{6}{|c|}{ Heptamers C } \\
\hline \multicolumn{6}{|c|}{$a_{c p}^{(C)}=6.0309063912$} \\
\hline \multicolumn{6}{|c|}{$b_{c p}^{(C)}=5.5371391576$} \\
\hline \multicolumn{6}{|c|}{$\phi_{0}=0.2843960475$} \\
\hline \multicolumn{6}{|c|}{$x_{1}=3.0154531956$} \\
\hline \multicolumn{6}{|c|}{$y_{1}=0.1230590461$} \\
\hline \multicolumn{6}{|c|}{$\phi_{3}=$} \\
\hline
\end{tabular}

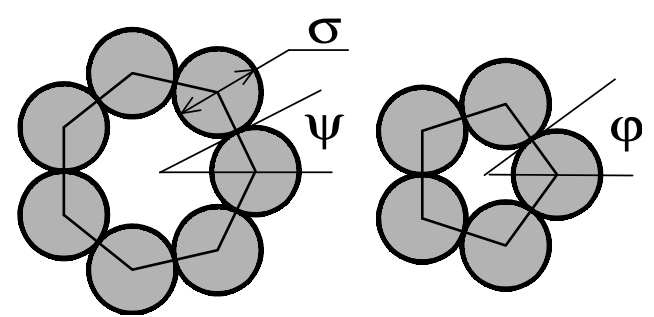

FIG. 1: Geometry of (a) the pentamer and (b) the heptamer 'molecules'. 
TABLE II: The equation of state and the unit cell parameters (extrapolated to the thermodynamic limit) of the studied structures of the pentamers and the heptamers. The parameters are defined as follows $v_{(\text {structure })}^{*}=V / V_{c p}^{(\text {structure })}, a_{(\text {structure })}^{*}=a / a_{c p}^{(\text {structure })}, b_{(\text {structure })}^{*}=b / b_{c p}^{(\text {structure })}, x_{i}^{*}=$ $\left(x_{i}-x_{0}\right) / a_{c p}^{(\text {structure })}, y_{i}^{*}=\left(y_{i}-y_{0}\right) / b_{c p}^{(\text {structure })}$, where $V$ is the (two-dimensional) volume (i.e. the area) of the system, $a, b$ are the sides of the (rectangular) unit cell, $V_{c p}^{(\text {structure })}, a_{c p}^{\text {(structure) }}, b_{c p}^{(\text {structure })}$ denote the close packing limits of $V, a, b$ for the structure $=P, A, B, C$, and $x_{i}, y_{i}, \phi_{i}$ are the average coordinates and orientations of the $i$-th molecule of the unit cell (see Fig.2). For the first three structures the values of $y_{1}^{*}, \phi_{0}, \phi_{1}$ are not shown because they are, respectively, equal to $a^{*} / 2,0, \pi / n$ (where $n=5$ for pentamers and $n=7$ for heptamers) within the experimental error.

\begin{tabular}{|c|c|c|c|c|}
\hline$p^{*}$ & 15 & 50 & 150 & 500 \\
\hline \multicolumn{5}{|c|}{ Pentamers $\mathrm{P}$} \\
\hline$\overline{v_{(P)}^{*}}$ & $1.04092(6)$ & $1.01196(1)$ & $1.003945(6)$ & $1.001178(4)$ \\
\hline$a_{(P)}^{*}$ & $1.03437(8)$ & $1.00934(2)$ & $1.003018(6)$ & $1.000985(4)$ \\
\hline$b_{(P)}^{*}$ & $1.00634(5)$ & $1.002594(7)$ & $1.000923(4)$ & $1.000283(3)$ \\
\hline$x_{1}^{*}$ & $0.44276(3)$ & $0.426830(7)$ & $0.423043(4)$ & $0.421886(3)$ \\
\hline \multicolumn{5}{|c|}{ Heptamers A } \\
\hline$\overline{v_{(A)}^{*}}$ & $1.02474(3)$ & $1.007188(8)$ & $1.002377(8)$ & $1.000712(3)$ \\
\hline$a_{(A)}^{*}$ & $1.01199(1)$ & $1.003485(5)$ & $1.001152(5)$ & $1.000345(2)$ \\
\hline$b_{(A)}^{*}$ & $1.01261(2)$ & $1.003664(5)$ & $1.001212(4)$ & $1.000363(2)$ \\
\hline$x_{1}^{*}$ & $0.62582(3)$ & $0.626170(7)$ & $0.626265(4)$ & $0.626298(2)$ \\
\hline \multicolumn{5}{|c|}{ Heptamers B } \\
\hline$\overline{v_{(B)}^{*}}$ & $1.02583(3)$ & $1.007217(8)$ & $1.002375(5)$ & $1.000710(4)$ \\
\hline$a_{(B)}^{*}$ & $1.01993(3)$ & $1.005569(7)$ & $1.001832(4)$ & $1.000547(3)$ \\
\hline$b_{(B)}^{*}$ & $1.00580(2)$ & $1.001620(4)$ & $1.000536(2)$ & $1.000160(2)$ \\
\hline$x_{1}^{*}$ & $0.46423(4)$ & $0.451666(6)$ & $0.448396(3)$ & $0.447272(3)$ \\
\hline \multicolumn{5}{|c|}{ Heptamers C } \\
\hline$v_{(C)}^{*}$ & $1.02470(5)$ & $1.00724(3)$ & $1.002402(3)$ & $1.0007193(9)$ \\
\hline$a_{(C)}^{*}$ & $1.01410(4)$ & $1.00413(2)$ & $1.001371(2)$ & $1.0004104(6)$ \\
\hline$b_{(C)}^{*}$ & $1.01047(2)$ & $1.00307(2)$ & $1.001018(2)$ & $1.0003049(4)$ \\
\hline$\phi_{0}$ & $0.28437(4)$ & $0.28439(3)$ & $0.284393(2)$ & $0.284395(1)$ \\
\hline$x_{1}$ & $0.50704(2)$ & $0.50206(1)$ & $0.500684(1)$ & $0.5002049(4)$ \\
\hline$y_{1}$ & $0.02368(2)$ & $0.022650(9)$ & $0.022366(1)$ & $0.0222666(3)$ \\
\hline$\phi_{1}$ & $-0.28437(5)$ & $-0.28439(3)$ & $-0.284393(3)$ & $-0.284395(1)$ \\
\hline$x_{2}$ & $0.21694(2)$ & $0.213997(8)$ & $0.213181(1)$ & $0.2128973(3)$ \\
\hline$y_{2}$ & $0.50523(2)$ & $0.501534(8)$ & $0.500509(1)$ & $0.5001524(3)$ \\
\hline$\phi_{2}$ & $0.16443(4)$ & $0.16441(3)$ & $0.164405(2)$ & $0.164403(2)$ \\
\hline$x_{3}$ & $0.72399(3)$ & $0.71606(2)$ & $0.713867(2)$ & $0.7131026(5)$ \\
\hline$y_{3}$ & $0.52891(3)$ & $0.52419(2)$ & $0.522875(2)$ & $0.5224191(4)$ \\
\hline$\phi_{3}$ & $-0.16443(5)$ & $-0.16441(3)$ & $-0.164405(2)$ & $-0.164403(2)$ \\
\hline
\end{tabular}


TABLE III: The dimensionless elastic compliances of the pentamer system in the rectangular solid phase. The dimensionless pressure is defined as: $p^{*}=p \sigma^{2} / k T$.

\begin{tabular}{|r|r|c|c|c|c|}
\hline \multicolumn{1}{|r|}{$N$} & $p^{*}$ & $S_{x x x x}^{*}$ & $S_{y y y y}^{*}$ & $S_{x x y y}^{*}$ & $S_{x y x y}^{*}$ \\
\hline 56 & 15 & $437(11) / 10^{5}$ & $280(5) / 10^{5}$ & $-231(7) / 10^{5}$ & $152(4) / 10^{5}$ \\
224 & 15 & $432(9) / 10^{5}$ & $278(7) / 10^{5}$ & $-231(5) / 10^{5}$ & $159(5) / 10^{5}$ \\
896 & 15 & $429(11) / 10^{5}$ & $270(7) / 10^{5}$ & $-225(8) / 10^{5}$ & $158(5) / 10^{5}$ \\
$\infty$ & 15 & $429(10) / 10^{5}$ & $272(7) / 10^{5}$ & $-227(7) / 10^{5}$ & $160(5) / 10^{5}$ \\
\hline 56 & 50 & $341(12) / 10^{6}$ & $186(3) / 10^{6}$ & $-145(6) / 10^{6}$ & $924(17) / 10^{7}$ \\
224 & 50 & $339(11) / 10^{6}$ & $183(4) / 10^{6}$ & $-140(6) / 10^{6}$ & $928(15) / 10^{7}$ \\
896 & 50 & $345(5) / 10^{6}$ & $176(2) / 10^{6}$ & $-134(2) / 10^{6}$ & $920(12) / 10^{7}$ \\
$\infty$ & 50 & $343(7) / 10^{6}$ & $178(3) / 10^{6}$ & $-135(3) / 10^{6}$ & $923(13) / 10^{7}$ \\
\hline 56 & 150 & $333(5) / 10^{7}$ & $185(2) / 10^{7}$ & $-127(2) / 10^{7}$ & $941(9) / 10^{8}$ \\
224 & 150 & $331(5) / 10^{7}$ & $179(3) / 10^{7}$ & $-120(2) / 10^{7}$ & $956(10) / 10^{8}$ \\
896 & 150 & $327(6) / 10^{7}$ & $179(3) / 10^{7}$ & $-118(3) / 10^{7}$ & $929(16) / 10^{8}$ \\
$\infty$ & 150 & $328(6) / 10^{7}$ & $178(3) / 10^{7}$ & $-118(3) / 10^{7}$ & $940(14) / 10^{8}$ \\
\hline 56 & 500 & $290(6) / 10^{8}$ & $162(2) / 10^{8}$ & $-109(3) / 10^{8}$ & $828(12) / 10^{9}$ \\
224 & 500 & $278(3) / 10^{8}$ & $160(2) / 10^{8}$ & $-105(2) / 10^{8}$ & $819(11) / 10^{9}$ \\
896 & 500 & $283(5) / 10^{8}$ & $162(2) / 10^{8}$ & $-104(3) / 10^{8}$ & $818(16) / 10^{9}$ \\
$\infty$ & 500 & $279(4) / 10^{8}$ & $161(2) / 10^{8}$ & $-104(3) / 10^{8}$ & $817(14) / 10^{9}$ \\
\hline
\end{tabular}


TABLE IV: The dimensionless elastic compliances of the heptamer system in the A phase. The symbols are the same as in Table III.

\begin{tabular}{|r|r|c|c|c|c|}
\hline \multicolumn{1}{|r|}{$N$} & $p^{*}$ & $S_{x x x x}^{*}$ & $S_{y y y y}^{*}$ & $S_{x x y y}^{*}$ & $S_{x y x y}^{*}$ \\
\hline 56 & 15 & $699(15) / 10^{6}$ & $746(13) / 10^{6}$ & $149(9) / 10^{6}$ & $201(7) / 10^{5}$ \\
224 & 15 & $682(10) / 10^{6}$ & $721(11) / 10^{6}$ & $160(7) / 10^{6}$ & $180(2) / 10^{5}$ \\
896 & 15 & $682(11) / 10^{6}$ & $706(9) / 10^{6}$ & $161(13) / 10^{6}$ & $180(2) / 10^{5}$ \\
$\infty$ & 15 & $679(10) / 10^{6}$ & $707(9) / 10^{6}$ & $163(10) / 10^{6}$ & $178(1) / 10^{5}$ \\
\hline 56 & 50 & $571(5) / 10^{7}$ & $615(6) / 10^{7}$ & $124(7) / 10^{7}$ & $114(2) / 10^{6}$ \\
224 & 50 & $570(6) / 10^{7}$ & $620(12) / 10^{7}$ & $139(5) / 10^{7}$ & $115(2) / 10^{6}$ \\
896 & 50 & $567(7) / 10^{7}$ & $601(11) / 10^{7}$ & $125(6) / 10^{7}$ & $109(2) / 10^{6}$ \\
$\infty$ & 50 & $568(7) / 10^{7}$ & $608(12) / 10^{7}$ & $128(6) / 10^{7}$ & $110(2) / 10^{6}$ \\
\hline 56 & 150 & $635(7) / 10^{8}$ & $696(7) / 10^{8}$ & $143(7) / 10^{8}$ & $1170(14) / 10^{8}$ \\
224 & 150 & $624(7) / 10^{8}$ & $690(12) / 10^{8}$ & $146(7) / 10^{8}$ & $1136(12) / 10^{8}$ \\
896 & 150 & $617(7) / 10^{8}$ & $664(11) / 10^{8}$ & $131(9) / 10^{8}$ & $1149(13) / 10^{8}$ \\
$\infty$ & 150 & $618(7) / 10^{8}$ & $671(12) / 10^{8}$ & $137(8) / 10^{8}$ & $1139(12) / 10^{8}$ \\
\hline 56 & 500 & $568(5) / 10^{9}$ & $619(5) / 10^{9}$ & $119(5) / 10^{9}$ & $1016(10) / 10^{9}$ \\
224 & 500 & $554(6) / 10^{9}$ & $599(9) / 10^{9}$ & $128(4) / 10^{9}$ & $1009(10) / 10^{9}$ \\
896 & 500 & $567(7) / 10^{9}$ & $588(6) / 10^{9}$ & $122(6) / 10^{9}$ & $998(12) / 10^{9}$ \\
$\infty$ & 500 & $560(10) / 10^{9}$ & $590(10) / 10^{9}$ & $126(5) / 10^{9}$ & $1000(12) / 10^{9}$ \\
\hline
\end{tabular}


TABLE V: The dimensionless elastic compliances of the heptamer system in the B phase. The symbols are the same as in Table III.

\begin{tabular}{|r|r|c|c|c|c|}
\hline \multicolumn{1}{|c|}{$N^{*}$} & $p^{*}$ & $S_{x x x x}^{*}$ & $S_{y y y y}^{*}$ & $S_{x x y y}^{*}$ & $S_{x y x y}^{*}$ \\
\hline 56 & 15 & $223(3) / 10^{5}$ & $892(19) / 10^{6}$ & $-631(18) / 10^{6}$ & $118(2) / 10^{5}$ \\
224 & 15 & $219(4) / 10^{5}$ & $861(7) / 10^{6}$ & $-606(12) / 10^{6}$ & $114(2) / 10^{5}$ \\
896 & 15 & $217(7) / 10^{5}$ & $848(11) / 10^{6}$ & $-622(22) / 10^{6}$ & $113(2) / 10^{5}$ \\
$\infty$ & 15 & $217(6) / 10^{5}$ & $847(8) / 10^{6}$ & $-612(18) / 10^{6}$ & $113(2) / 10^{5}$ \\
\hline 56 & 50 & $129(2) / 10^{6}$ & $607(8) / 10^{7}$ & $-216(8) / 10^{7}$ & $777(5) / 10^{7}$ \\
224 & 50 & $126(2) / 10^{6}$ & $584(5) / 10^{7}$ & $-200(9) / 10^{7}$ & $768(13) / 10^{7}$ \\
896 & 50 & $125(2) / 10^{6}$ & $577(5) / 10^{7}$ & $-196(10) / 10^{7}$ & $731(12) / 10^{7}$ \\
$\infty$ & 50 & $125(2) / 10^{6}$ & $576(5) / 10^{7}$ & $-195(11) / 10^{7}$ & $747(6) / 10^{7}$ \\
\hline 56 & 150 & $133(2) / 10^{7}$ & $650(6) / 10^{8}$ & $-195(10) / 10^{8}$ & $826(9) / 10^{8}$ \\
224 & 150 & $133(1) / 10^{7}$ & $636(4) / 10^{8}$ & $-183(10) / 10^{8}$ & $799(7) / 10^{8}$ \\
896 & 150 & $132(2) / 10^{7}$ & $641(9) / 10^{8}$ & $-188(12) / 10^{8}$ & $807(11) / 10^{8}$ \\
$\infty$ & 150 & $132(2) / 10^{7}$ & $637(7) / 10^{8}$ & $-184(11) / 10^{8}$ & $800(9) / 10^{8}$ \\
\hline 56 & 500 & $117(2) / 10^{8}$ & $580(6) / 10^{9}$ & $-158(8) / 10^{9}$ & $714(7) / 10^{9}$ \\
224 & 500 & $117(2) / 10^{8}$ & $572(6) / 10^{9}$ & $-157(6) / 10^{9}$ & $687(9) / 10^{9}$ \\
896 & 500 & $116(2) / 10^{8}$ & $581(9) / 10^{9}$ & $-155(9) / 10^{9}$ & $685(9) / 10^{9}$ \\
$\infty$ & 500 & $116(2) / 10^{8}$ & $576(8) / 10^{9}$ & $-156(8) / 10^{9}$ & $681(9) / 10^{9}$ \\
\hline
\end{tabular}


TABLE VI: The dimensionless elastic compliances of the heptamer system in the $\mathrm{C}$ phase. The symbols are the same as in Table III.

\begin{tabular}{|r|r|c|c|c|c|}
\hline$N$ & $p^{*}$ & $S_{x x x x}^{*}$ & $S_{y y y}^{*}$ & $S_{x x y y}^{*}$ & $S_{x y x y}^{*}$ \\
\hline 36 & 15 & $981(18) / 10^{6}$ & $661(11) / 10^{6}$ & $44(10) / 10^{6}$ & $774(10) / 10^{6}$ \\
144 & 15 & $964(25) / 10^{6}$ & $663(15) / 10^{6}$ & $63(10) / 10^{6}$ & $744(13) / 10^{6}$ \\
224 & 15 & $920(8) / 10^{6}$ & $649(4) / 10^{6}$ & $52(8) / 10^{6}$ & $736(8) / 10^{6}$ \\
576 & 15 & $928(15) / 10^{6}$ & $645(6) / 10^{6}$ & $49(9) / 10^{6}$ & $744(7) / 10^{6}$ \\
896 & 15 & $928(16) / 10^{6}$ & $658(12) / 10^{6}$ & $47(9) / 10^{6}$ & $729(14) / 10^{6}$ \\
$\infty$ & 15 & $927(15) / 10^{6}$ & $652(11) / 10^{6}$ & $53(8) / 10^{6}$ & $733(11) / 10^{6}$ \\
\hline 36 & 50 & $785(6) / 10^{7}$ & $594(4) / 10^{7}$ & $51(4) / 10^{7}$ & $600(4) / 10^{7}$ \\
144 & 50 & $787(15) / 10^{7}$ & $576(7) / 10^{7}$ & $69(6) / 10^{7}$ & $574(9) / 10^{7}$ \\
224 & 50 & $766(7) / 10^{7}$ & $565(6) / 10^{7}$ & $67(7) / 10^{7}$ & $567(5) / 10^{7}$ \\
576 & 50 & $741(12) / 10^{7}$ & $562(7) / 10^{7}$ & $57(6) / 10^{7}$ & $581(5) / 10^{7}$ \\
896 & 50 & $751(13) / 10^{7}$ & $575(6) / 10^{7}$ & $58(8) / 10^{7}$ & $590(12) / 10^{7}$ \\
$\infty$ & 50 & $755(13) / 10^{7}$ & $566(7) / 10^{7}$ & $63(8) / 10^{7}$ & $576(9) / 10^{7}$ \\
\hline 36 & 150 & $834(6) / 10^{8}$ & $643(7) / 10^{8}$ & $56(5) / 10^{8}$ & $631(6) / 10^{8}$ \\
144 & 150 & $813(26) / 10^{8}$ & $627(14) / 10^{8}$ & $70(8) / 10^{8}$ & $617(12) / 10^{8}$ \\
224 & 150 & $830(10) / 10^{8}$ & $650(10) / 10^{8}$ & $78(5) / 10^{8}$ & $616(7) / 10^{8}$ \\
576 & 150 & $819(11) / 10^{8}$ & $625(8) / 10^{8}$ & $74(5) / 10^{8}$ & $624(7) / 10^{8}$ \\
896 & 150 & $830(10) / 10^{8}$ & $620(10) / 10^{8}$ & $51(7) / 10^{8}$ & $609(10) / 10^{8}$ \\
$\infty$ & 150 & $822(15) / 10^{8}$ & $628(11) / 10^{8}$ & $60(7) / 10^{8}$ & $614(10) / 10^{8}$ \\
\hline 36 & 500 & $770(12) / 10^{9}$ & $579(13) / 10^{9}$ & $65(5) / 10^{9}$ & $558(6) / 10^{9}$ \\
144 & 500 & $736(10) / 10^{9}$ & $559(10) / 10^{9}$ & $68(8) / 10^{9}$ & $543(5) / 10^{9}$ \\
224 & 500 & $754(12) / 10^{9}$ & $558(8) / 10^{9}$ & $62(4) / 10^{9}$ & $552(6) / 10^{9}$ \\
576 & 500 & $749(6) / 10^{9}$ & $573(5) / 10^{9}$ & $67(4) / 10^{9}$ & $561(5) / 10^{9}$ \\
896 & 500 & $751(9) / 10^{9}$ & $565(9) / 10^{9}$ & $60(9) / 10^{9}$ & $562(7) / 10^{9}$ \\
$\infty$ & 500 & $745(9) / 10^{9}$ & $562(8) / 10^{9}$ & $64(7) / 10^{9}$ & $566(7) / 10^{9}$ \\
\hline
\end{tabular}


TABLE VII: Comparison of results obtained for $N=56$ pentamers at the dimensionless pressure $p^{*}=50$ : (a) a single long run, (b) 16 parallel runs initialized from various structures generated in the long run,(c) for 16 parallel runs initialized from a periodic lattice obtained by uniform scaling of the close packed structure. Details of the runs are discussed in text.

\begin{tabular}{|c|c|c|c|c|}
\hline run & $S_{x x x x}^{*}$ & $S_{y y y y}^{*}$ & $S_{x x y y}^{*}$ & $S_{x y x y}^{*}$ \\
\hline$(\mathrm{a})$ & $3365(15) / 10^{7}$ & $1835(8) / 10^{7}$ & $-1385(7) / 10^{7}$ & $960(7) / 10^{7}$ \\
(b) & $3363(16) / 10^{7}$ & $1823(7) / 10^{7}$ & $-1385(9) / 10^{7}$ & $947(6) / 10^{7}$ \\
(c) & $3328(22) / 10^{7}$ & $1835(6) / 10^{7}$ & $-1370(8) / 10^{7}$ & $953(6) / 10^{7}$ \\
\hline
\end{tabular}

TABLE VIII: The extrapolated values of the rescaled (by the free volume bulk modulus) elastic constants and directional Poisson ratios in the close packing limit for the pentamers and heptamers. The errors are between two and four percent. $P$ denotes the pentamers, whereas $\mathrm{A}, \mathrm{B}$, and $\mathrm{C}$ denote the studied phases of the heptamers. It is worth to notice that $C_{x x y y}$ is negative for the structures $\mathrm{A}$ and $\mathrm{C}$. This implies negative values of the directional Poisson ratios shown in the last two rows.

\begin{tabular}{|c|c|c|c|c|}
\hline & $P$ & $A$ & $B$ & $C$ \\
\hline$C_{x x x x} / B_{T}^{F V}$ & 1.065 & 2.697 & 1.284 & 1.983 \\
\hline$C_{y y y y} / B_{T}^{F V}$ & 1.953 & 2.510 & 2.596 & 2.594 \\
\hline$C_{x x y y} / B_{T}^{F V}$ & 0.695 & -0.565 & 0.320 & -0.208 \\
\hline$C_{x y x y} / B_{T}^{F V}$ & 0.731 & 0.360 & 0.517 & 0.648 \\
\hline$\nu_{x y}$ & 0.352 & -0.222 & 0.123 & -0.084 \\
\hline$\nu_{y x}$ & 0.660 & -0.207 & 0.239 & -0.109 \\
\hline
\end{tabular}




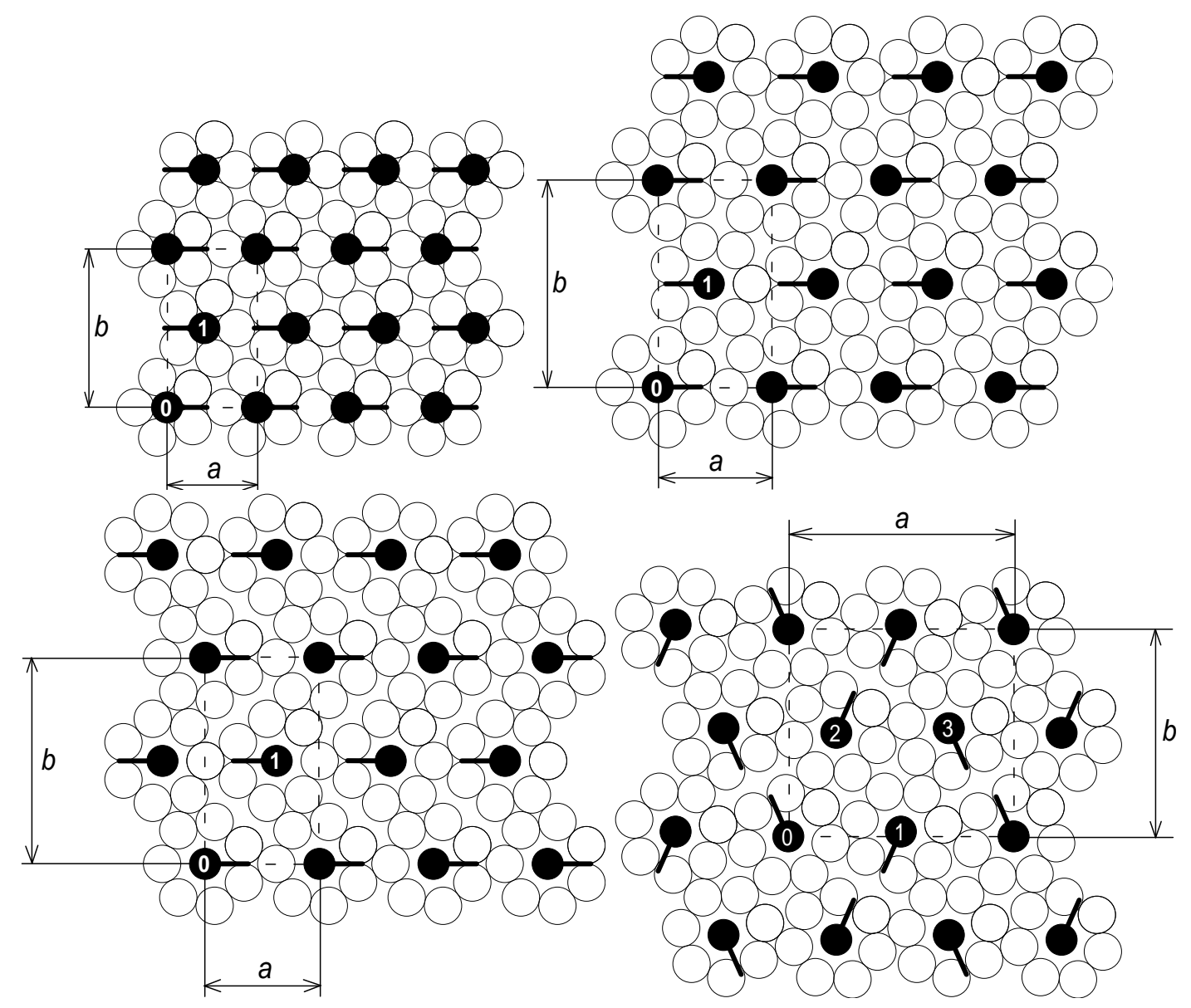

FIG. 2: The close packed structures in the (a) pentamer and (b)-(d) heptamer systems. The unit cell for each of these structures is rectangular. In the case of the heptamers they are further referred to as (b) the B-structure, (c) the A-structure (which is denser than the B-structure at close packing), (d) the C-structure (which is the most dense structure of the known heptamer structures at close packing [55, 58]). The molecular orientations, $\phi$, are indicated in this figure by line intervals of orientations $5 \phi$ for pentamers and $7 \phi$ for heptamers. 


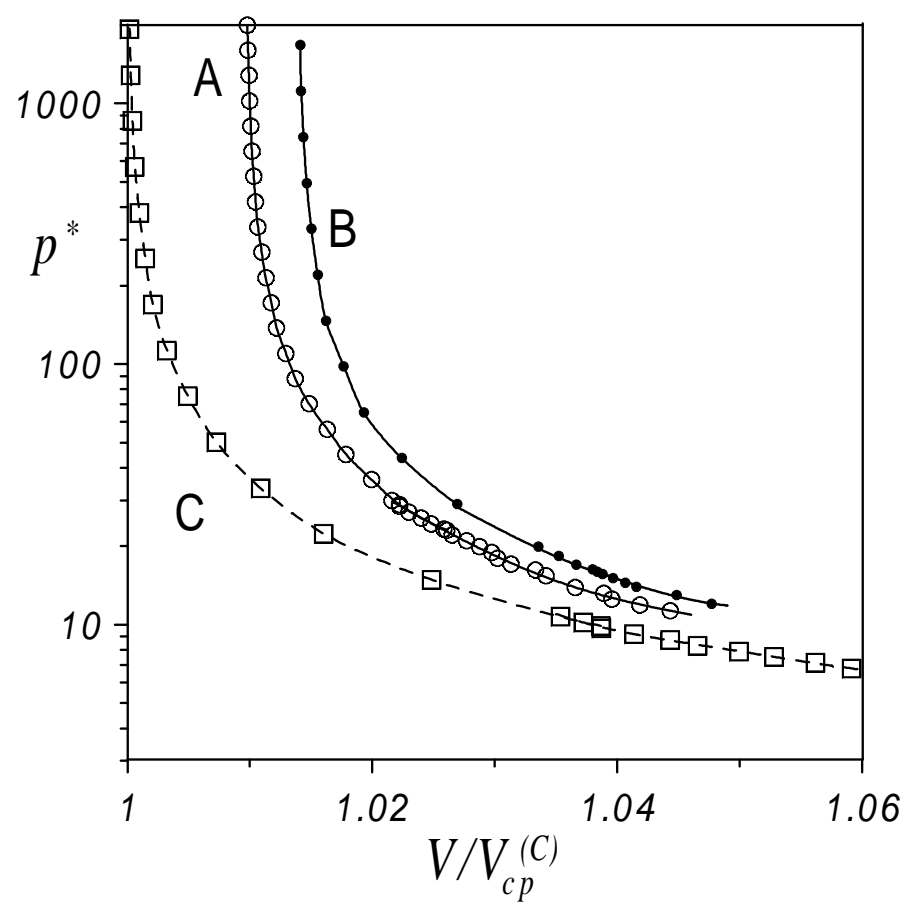

FIG. 3: The izotherms of dense solid structures for the heptamer system. $v^{*}=V / V_{c p}^{(C)}$ where $V$ is the two-dimensional volume (i.e. the area) of the system, and $V_{c p}^{(C)}$ denotes its value for the structure $C$ at close packing; $p^{*}$ is the dimensionless pressure defined in Table III. It can be seen that the isotherm corresponding to the B-structure is located above the izotherm of the A-structure, which, in turn, is located above the isotherm of the C-structure [55, 58]. 

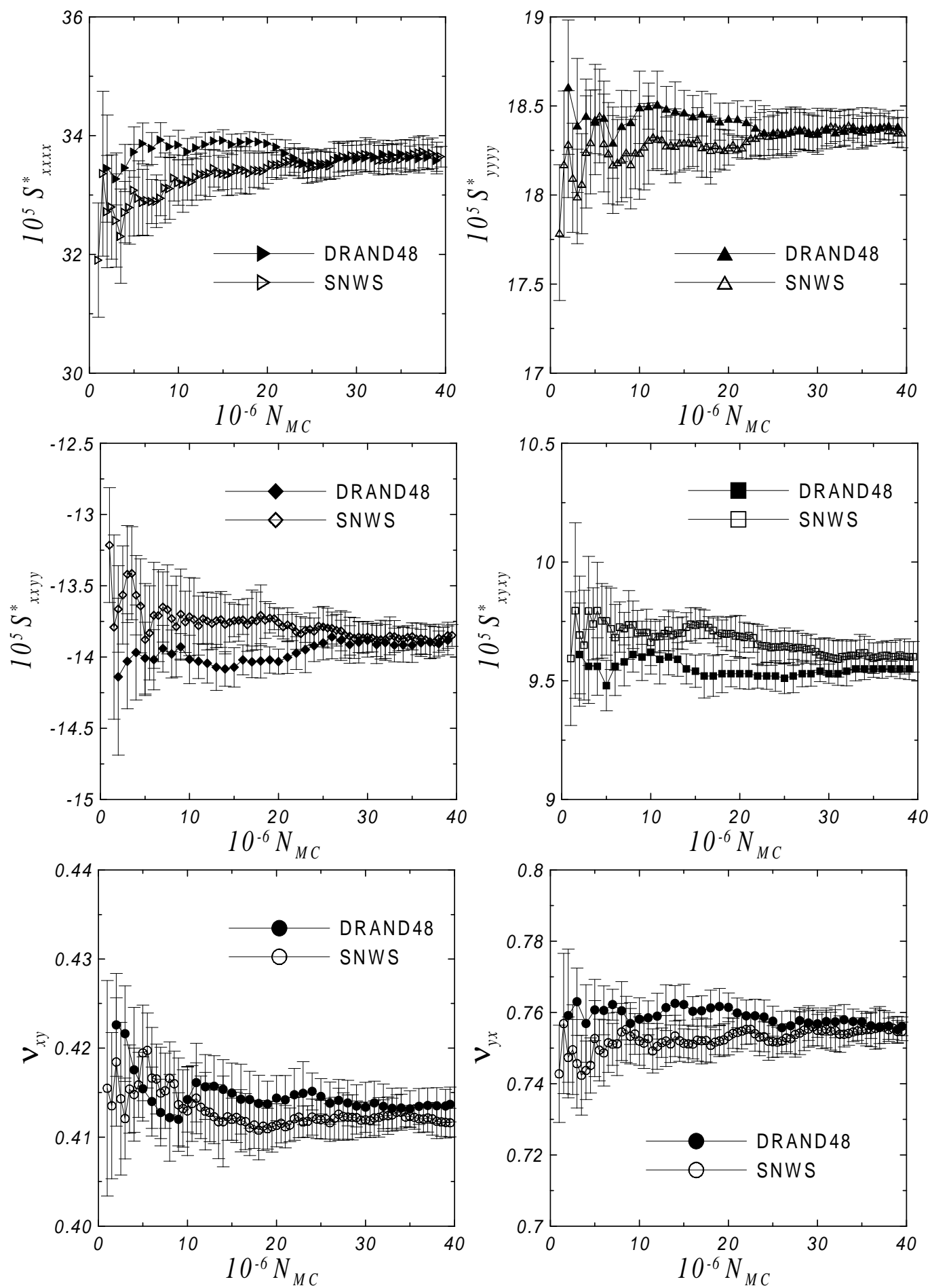

FIG. 4: An example of the accumulation plots of (a)-(d) the dimensionless elastic compliances and (e)-(f) the Poisson ratios obtained for $N=56$ pentamers in the rectangular phase at $p^{*}=50$ simulated by using two different random number generators $D R A N D 48$ (full symbols) and $S N W S$ (open symbols). 

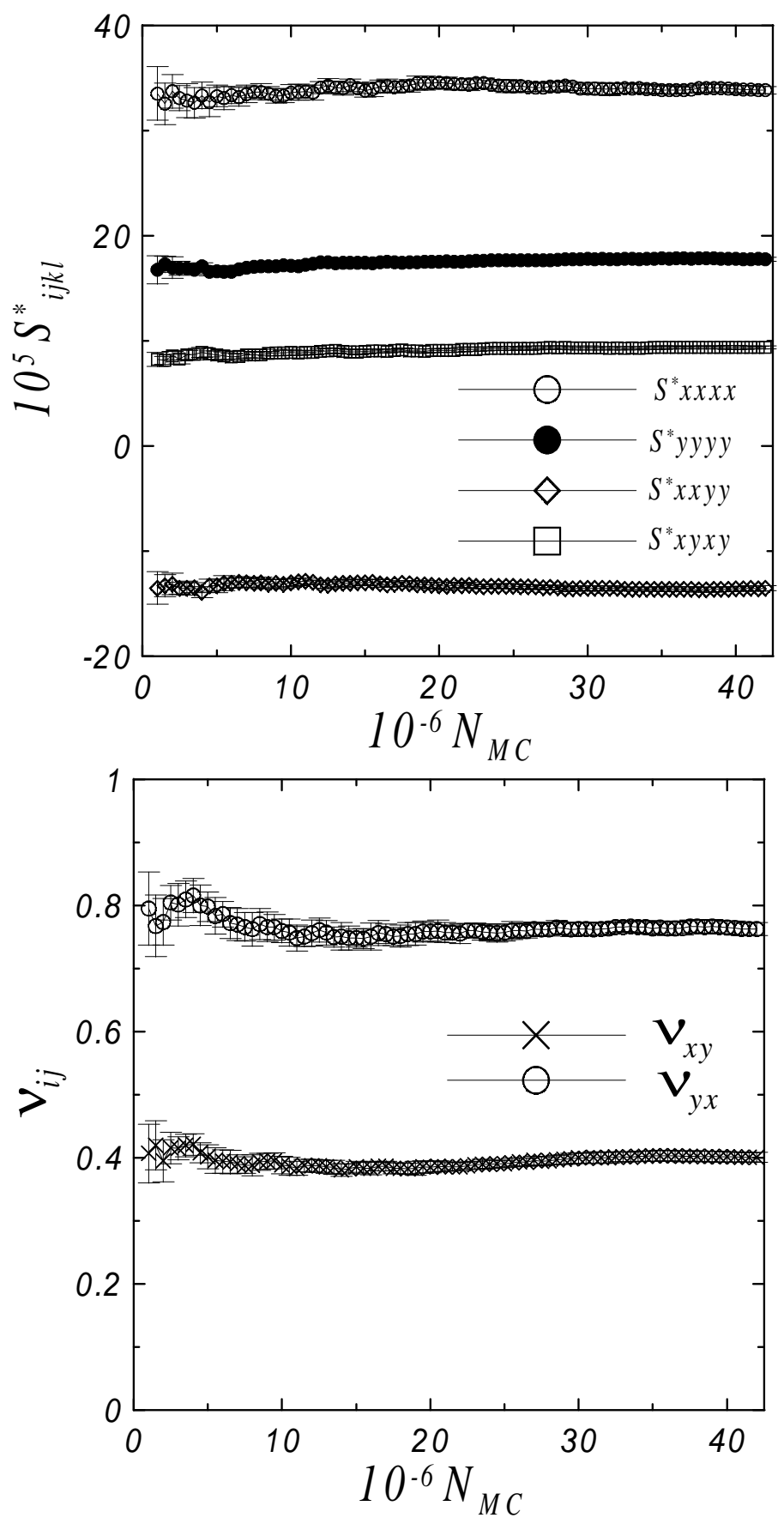

FIG. 5: The accumulation plots of (a) the dimensionless elastic compliances and (b) the Poisson ratios obtained for $N=896$ pentamers in the rectangular phase at $p^{*}=50$. 

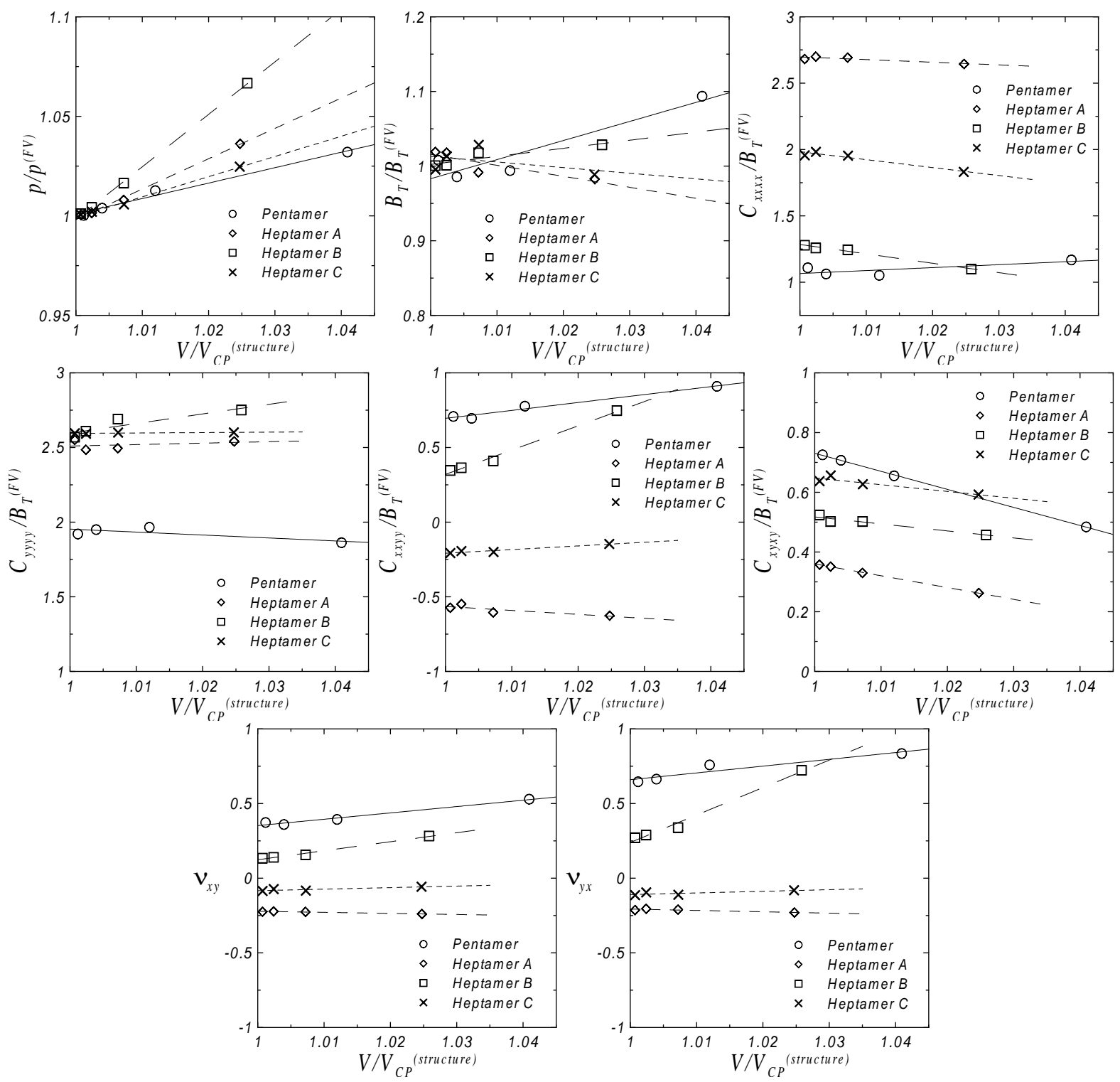

FIG. 6: The experimental data obtained for the pentamers (open circles) and the heptamers (the phases $\mathrm{A}, \mathrm{B}$ and $\mathrm{C}$ are represented by diamonds, squares, and crosses, respectively) divided by: their asymptotics obtained in frames of the free volume approximation for (a) the pressure and (b) the bulk modulus, and by the free volume estimate of the bulk modulus for (c)-(f) the elastic constants. The elastic constants, $C_{i j k l}$, were calculated by the formulae (7) and (4). In (g) and (h) we show the Poisson ratios obtained from (10). 